Review Article

\title{
Digital Rectal Examination and Balloon Expulsion Test in the Study of Defecatory Disorders: Are They Suitable as Screening or Excluding Tests?
}

\author{
Ana C. Caetano, ${ }^{1,2,3}$ André Santa-Cruz, ${ }^{2,3,4}$ and Carla Rolanda ${ }^{1,2,3}$ \\ ${ }^{1}$ Department of Gastroenterology, Braga Hospital, Braga, Portugal \\ ${ }^{2}$ Life and Health Sciences Research Institute (ICVS), School of Health Sciences, University of Minho, Braga, Portugal \\ ${ }^{3}$ ICVS/3B's-PT Government Associate Laboratory, Guimarães, Braga, Portugal \\ ${ }^{4}$ Department of Internal Medicine, Braga Hospital, Braga, Portugal
}

Correspondence should be addressed to Ana C. Caetano; anaceliacaetanocs@gmail.com

Received 4 March 2016; Accepted 22 September 2016

Academic Editor: Přemysl Berčík

Copyright (c) 2016 Ana C. Caetano et al. This is an open access article distributed under the Creative Commons Attribution License, which permits unrestricted use, distribution, and reproduction in any medium, provided the original work is properly cited.

Background. Rome III criteria add physiological criteria to symptom-based criteria of chronic constipation (CC) for the diagnosis of defecatory disorders (DD). However, a gold-standard test is still lacking and physiological examination is expensive and timeconsuming. Aim. Evaluate the usefulness of two low-cost tests-digital rectal examination (DRE) and balloon expulsion test (BET) - as screening or excluding tests of DD. Methods. We performed a systematic search in PUBMED and MEDLINE. We selected studies where constipated patients were evaluated by DRE or BET. Heterogeneity was assessed and random effect models were used to calculate the sensitivity, specificity, and negative predictive value (NPV) of the DRE and the BET. Results. Thirteen studies evaluating BET and four studies evaluating DRE (2329 patients) were selected. High heterogeneity $\left(I^{2}>80 \%\right)$ among studies was demonstrated. The studies evaluating the BET showed a sensitivity and specificity of $67 \%$ and $80 \%$, respectively. Regarding the DRE, a sensitivity of $80 \%$ and specificity of $84 \%$ were calculated. NPV of $72 \%$ for the BET and NPV of $64 \%$ for the DRE were estimated. The sensitivity and specificity were similar when we restrict the analysis to studies using Rome criteria to define CC. The BET seems to perform better when a cut-off time of 2 minutes is used and when it is compared with a combination of physiological tests. Considering the DRE, strict criteria seem to improve the sensitivity but not the specificity of the test. Conclusion. Neither of the low-cost tests seems suitable for screening or excluding DD.

\section{Introduction}

Defecatory disorders (DD) are common in the community with an incidence of 22 per 100,000 person years [1]. DD may result from disordered anorectal function (e.g., inadequate rectal propulsive forces and/or increased resistance to evacuation) or rectal structural disturbances (which prevent its reservoir function); these pathophysiological mechanisms may coexist [2-7]. Rome III criteria [8] provide a symptombased definition of chronic constipation (CC) and recognize subgroups of CC based on both symptoms and physiological criteria. According to Rome III classification, the diagnosis of DD is established when a patient with CC has evidence of at least two physiological impairments in any of the anorectal tests-balloon expulsion test (BET), imaging, anorectal manometry (ARM), or electromyography (EMG). Rome III diagnostic criteria fulfilled for the last 3 months with symptom onset at least 6 months prior to diagnosis for functional defecation disorders are as follows:

(1) The patient must satisfy diagnostic criteria for functional constipation.

(2) During repeated attempts to defecate the patient must have at least two of the following:

(a) Evidence of impaired evacuation, based on BET or imaging. 
(b) Inappropriate contraction of the pelvic floor muscles (i.e., anal sphincter, or puborectalis) or less than $20 \%$ relaxation of basal resting sphincter pressure by ARM, imaging, or EMG.

(c) Inadequate propulsive forces assessed by ARM or imaging. follows:

Diagnostic criteria for functional constipation are as

(1) The patient must include two or more of the following: (a) straining during at least $25 \%$ of defecations, (b) lumpy or hard stools at least $25 \%$ of defecations, (c) sensation of incomplete evacuation at least $25 \%$ of defecations, (d) sensation of anorectal obstruction/ blockage at least $25 \%$ of defecations, (e) manual maneuvers to facilitate at least $25 \%$ of defecations (e.g., digital evacuation, support of the pelvic floor), and (f) fewer than three defecations per week.

(2) Loose stools are rarely present without the use of laxatives.

(3) There are insufficient criteria for irritable bowel syndrome.

Based on evidence that shows substantial symptom overlap among the different pathophysiological entities of CC [9-11], Rome III criteria state the need of anorectal tests to substantiate the diagnosis of DD. An extensive technical description of these tests is made elsewhere $[6,12]$.

However, the findings on these different tests may not be in agreement $[11,13]$. Thirty percent of patients with marked evacuatory symptoms have negative tests for DD [11] and results compatible with DD are documented in around $25 \%$ of healthy individuals [14-18]. Most authors from tertiary centres emphasize the phenotypic heterogeneity of DD $[19,20]$ and the unhelpful settings in which these tests are performed [21] to explain this disagreement. However, they tend to trust the potential benefit of an extensive and ultimately enhanced diagnostic approach $[7,22]$. Another aspect to take into account is the cost involved in diagnosing DD. The exact impact of CC diagnostic assessment in Western Europe healthcare systems is unknown [23] but an American study by Rantis Jr. et al. [24] reports a costly work-up without even considering the indirect cost [25].

A low-cost screening option to identify DD in clinical settings with limited resources would have clinical [26] and financial advantages.

The digital rectal examination (DRE) is the simplest and cheapest clinical tool for identifying DD, thoroughly described by Talley [27]. Tantiphlachiva et al. [28] proposed that DD could be diagnosed by DRE, if two of the following criteria were present: (1) a paradoxical anal contraction or impaired anal relaxation, (2) impaired push effort, and (3) absence of perineal descent. Orkin et al. [29] compared a semiquantitative DRE scoring system (DRESS) and found it reasonably accurate in comparison to ARM for assessing anal resting tone and squeeze function and for identifying dyssynergia. Due to the emphasis given nowadays to technology rather than to clinical skills [30], this simple tool may sometimes be underused [29].
The BET is a simple procedure, first described by Preston and Lennard-Jones [2], that evaluates a patient's ability to evacuate a filled balloon. Different methodologies consider air filled or water-filled balloon and the lying or seated position to perform the BET. Recommended time values range from less than 1 minute to up to 5 minutes [31]. Recent studies showed that the inability to expel the balloon is suggestive of DD [16, 32]. Interestingly, Minguez et al. [33] proposed that a normal test would exclude DD. Contradictory data showed that some patients with DD could expel the balloon [11, 19, 34] making this test alone apparently insufficient to exclude a diagnosis of DD.

Chiarioni et al. [34] addressed the issue of BET reproducibility and found a perfect reproducibility in 280 patients with constipation ( $98 \%$, cut-off $<2$ minutes), adding value to this attractive screening option.

Our aim was to conduct a systematic review of the studies that included patients with CC who performed a lowcost anorectal evaluation (DRE or BET) to determine the sensitivity and specificity of these tests for the diagnosis of $\mathrm{DD}$ and their potential value as screening or excluding tests.

\section{Method}

We conducted a literature search in the online databases MEDLINE and PUBMED. The search terms included chronic constipation, functional constipation, defecatory disorders, dyssynergic defecation, dyssynergic evacuation, pelvic floor dysfunction, pelvic floor dyssynergia, balloon expulsion test, balloon evacuation, digital rectal examination, and digital rectal exploration. Besides, a manual search of the references from previously published systematic reviews was also performed to identify additional studies of interest. The search was limited to manuscripts published in English between 1975 and 2015 in adult population. Abstracts were screened and potentially relevant articles were reviewed.

2.1. Selection of Studies and Data Extraction. We selected studies where constipated patients were evaluated by DRE or BET. The definition of constipation is not clearly defined in most articles so we considered any study evaluating patients with constipation. For analysis we included only the studies in which (i) patients were unselected so they did not have a defined subtype of constipation, (ii) criteria for DD were clearly stated and comparative physiological test was defined by the author, and (iii) the data of the manuscript was sufficient to determine sensitivity, specificity, and NPV for the DRE or the BET. Manuscripts with a study population with less than 25 patients were excluded.

Caetano and Rolanda extracted the data. The inclusion criteria and the extracted data are presented in Table 1.

2.2. Meta-Analysis and Subgroup Analyses. The sensitivity, specificity, and NPV of the BET and the DRE were calculated for the included studies and presented in forest plots. Random effects models were used to provide pooled estimates of sensitivity and specificity and their corresponding $95 \%$ confidence intervals (CI). Statistical heterogeneity among the 


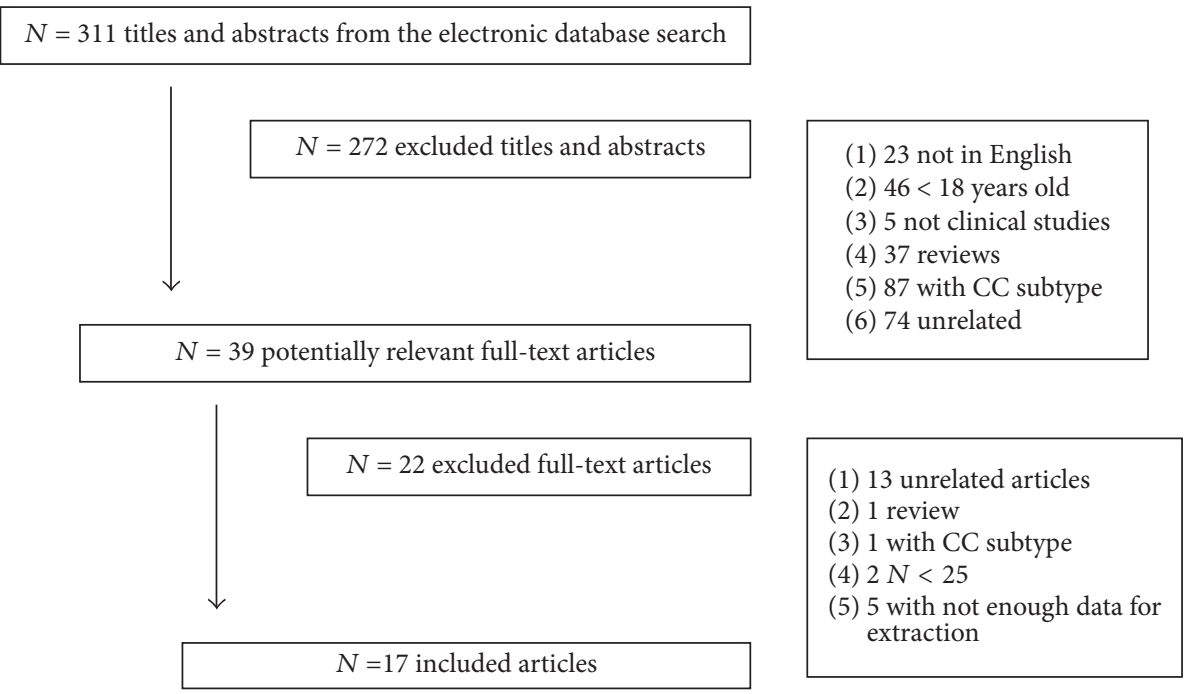

FIGURE 1: Flowchart of the literature search and study selection process.

TABLE 1: Inclusion criteria and extracted data.

\begin{tabular}{ll}
\hline & $N>25$ \\
& Patients with chronic constipation (no strict criteria) \\
& Patients with no specified subtype of chronic \\
Studies & constipation \\
& Performance of DRE or BET (in comparison to a \\
& defined physiological test) \\
& Sufficient data to calculate sensitivity, specificity, \\
& NPV \\
\hline & Gender distribution \\
& Mean age \\
& DRE and BET characteristics \\
& Criteria for positivity in DRE and BET \\
& Comparative anorectal physiological test \\
& Criteria for diagnosis of defecatory disorder in \\
physiological test & Number of true positive, true negative, false positive, \\
false negative
\end{tabular}

DRE: digital rectal examination. BET: balloon expulsion test.

studies was investigated using $I^{2}$ statistic. All analyses were performed using the Comprehensive Meta-Analysis version 3 (Biostat, Englewood, NJ, USA).

\section{Results}

3.1. Eligible Studies. With the electronic database and manual search, a total of 311 titles and abstracts were screened and 39 full-text manuscripts were reviewed. Seventeen articles fulfilled the criteria and were included for the analysis. We present the graphic flow in Figure 1. The included articles were published from 1987 until 2015. We used data regarding the BET in 13 studies and data regarding the DRE in 4 studies. The clinical series varied between 32 and 295 patients completing a total of 2329 patients. The data from the majority of studies (88\%) were collected prospectively. Rome criteria for CC were used in 7 studies. The combined mean age of the patients was
47,5 years and $75 \%$ were women (excluding 3 studies that did not describe gender distribution).

3.2. Balloon Expulsion Test. Thirteen studies met our inclusion criteria and supplied sufficient data to determine sensitivity, specificity, and NPV for the BET.

The meta-analysis showed high heterogeneity $\left(I^{2}=90 \%\right)$ among these 13 studies. Pooled sensitivity (95\% CI) for failed or impaired balloon expulsion by any criteria was $67 \%$ (CI $53-79 \%$ ) and pooled specificity was $80 \%$ (CI 73-86\%). The sensitivity and the specificity did not change when we limited the analysis to the studies with Rome criteria for CC (5 studies, sensitivity of $65 \%$ and specificity of $78 \%$ ). Table 2 summarizes the data related to the BET and Figures 2 and 3 show the forest plot for the sensitivity and the specificity of the BET.

Taking into consideration the characteristics of the BET, when we evaluate the studies that used only water as simulated stool (10 studies), sensitivity was $71 \%$ and specificity was $80 \%$. In the evaluation of studies that used a volume $\geq 50 \mathrm{~mL}$ or the seated position (11 and 12 studies, resp.), the sensitivity was $67 \%$ and the specificity was $80 \%$. When we performed the subanalysis of the BET with normal time to evacuate $\leq$ 2 minutes (4 studies), sensitivity rose to $74 \%$ (CI $47-90 \%$ ) and the specificity rose to $84 \%$ (CI 71-92\%). The subanalysis of the gold-standard comparative test showed sensitivity and specificity of the BET of $72 \%$ and $81 \%$ in comparison to ARM (5 studies), $53 \%$ and $77 \%$ in comparison to defecography (4 studies), and $88 \%$ and $85 \%$ in comparison to a combination of tests (2 studies $[33,35])$. The pooled NPV of the BET was $72 \%$ (CI 63-80\%).

3.3. Digital Rectal Examination. Four studies considering the DRE were included after careful evaluation. Once more, high heterogeneity between studies was established by the metaanalysis $\left(I^{2}=91 \%\right)$. The pooled sensitivity (95\% CI) for the diagnosis of DD with the DRE using any criteria was $80 \%$ (CI 
TABLE 2: Summary of studies with balloon expulsion test.

\begin{tabular}{|c|c|c|c|c|c|c|c|c|c|}
\hline Study reference & $N$ & $\begin{array}{l}\text { Gender } \\
\text { (fem) }\end{array}$ & $\begin{array}{l}\text { Age } \\
(\mathrm{y})\end{array}$ & $\begin{array}{l}\text { BET (simulated stool, } \\
\text { volume }(\mathrm{mL}), \text { body } \\
\text { position, time }(\mathrm{min}))\end{array}$ & $\begin{array}{l}\text { Comparative test } \\
\text { (used criteria) }\end{array}$ & $\mathrm{Se}$ & $\mathrm{Sp}$ & PPV & NPV \\
\hline [39] & 61 & 61 & 50 & W 50 S 2 & $\begin{array}{l}\text { HRM and/or DEF } \\
\text { pr-pc or pr-nr, ce-i }\end{array}$ & 33 & 80 & 71 & 45 \\
\hline$[34]$ & 236 & 264 & 44 & W 50 S 2 & ARM pr-pc or pr-nr & 85 & 71 & 77 & 80 \\
\hline [11] & 100 & 80 & 53 & W 50 S 3 & ARM pr-pc & 60 & 1 & 100 & 52 \\
\hline$[5]$ & 52 & 49 & 35 & W 50 S 5 & ARM pr-pc or pr-nr & 94 & 75 & 89 & 86 \\
\hline [40] & 295 & 295 & 48 & W 50 S 3 & HRM pr-pc or pr-nr & 29 & 78 & 39 & 70 \\
\hline$[41]$ & 132 & 256 & 52 & A 50 S 2 & ARM pr-pc or pr-nr & 76 & 92 & 92 & 80 \\
\hline$[33]$ & 130 & 124 & 38 & W SD S 1 & $\begin{array}{c}\text { ARM + DEF pr-pc or } \\
\text { pr-nr, ce-i }\end{array}$ & 88 & 89 & 64 & 97 \\
\hline$[42]$ & 46 & 34 & 46 & A $20 \mathrm{~S}$ & DEF ce-i & 41 & 92 & 82 & 63 \\
\hline$[43]$ & 134 & 112 & 52 & W $60 \mathrm{~S}$ & DEF ce-i or ce-f & 37 & 88 & 71 & 64 \\
\hline$[45]$ & 74 & 65 & & W 10 & DEF ce-i or ce-f & 87 & 63 & 51 & 91 \\
\hline$[14]$ & 32 & & 46 & W 50 & EMG i & 75 & 88 & 95 & 54 \\
\hline$[35]$ & 35 & 30 & 44 & W 50 S 5 & $\begin{array}{c}\mathrm{ARM}+\mathrm{DEF} / \mathrm{CTT} \\
\text { pr-pc or pr-nr, ce-i, stt }\end{array}$ & 89 & 76 & 80 & 87 \\
\hline [13] & 123 & 118 & 51 & A $60 \mathrm{~S} 5$ & DEF ce-i & 52 & 58 & 57 & 54 \\
\hline
\end{tabular}

BET: balloon expulsion test, HRM: high resolution manometry, ARM: anorectal manometry, DEF: defecography, EMG: electromyography, and CCT: colonic transit time.

BET: W: water, A: air, SD: sustained desire to evacuate, and S: seated position.

HRM or ARM: pr-pc (puborectalis paradoxical contraction) or pr-nr (puborectalis nonrelaxing).

DEF: ce-i (contrast evacuation impaired) or ce-f (contrast evacuation failed in $30 \mathrm{~s}$ ).

EMG: i (activity increased).

CTT: stt (slow transit time).

TABLE 3: Summary of studies with digital rectal examination.

\begin{tabular}{|c|c|c|c|c|c|c|c|c|c|}
\hline $\begin{array}{l}\text { Study } \\
\text { reference }\end{array}$ & $N$ & $\begin{array}{l}\text { Gender } \\
\text { (fem) }\end{array}$ & $\begin{array}{l}\text { Age } \\
\text { (y) }\end{array}$ & $\begin{array}{c}\text { DRE } \\
\text { (used criteria) }\end{array}$ & $\begin{array}{l}\text { Comparative test } \\
\text { (used criteria) }\end{array}$ & $\mathrm{Se}$ & Sp & PPV & NPV \\
\hline$[37]$ & 268 & 152 & 64 & $\begin{array}{c}2 \text { of as-pc/as-nr, pe-i, } \\
\text { pd-a }\end{array}$ & HRM (type I-IV DD) & 93 & 59 & 91 & 66 \\
\hline [28] & 209 & 18 & 41 & $\begin{array}{c}2 \text { of as-pc/as-nr, } \\
\text { am-nc, pd-a }\end{array}$ & $\begin{array}{l}\text { ARM + BET or CTT } \\
\text { pr-p or pr-nr, ne, stt }\end{array}$ & 73 & 85 & 97 & 31 \\
\hline [36] & 168 & & 44 & pr-pc & $\mathrm{ARM}+\mathrm{CTT}$ & 83 & 95 & 98 & 65 \\
\hline [47] & 136 & & & pr-pc & $\mathrm{DEF}+\mathrm{EMG}$ aa-ni, i & 58 & 88 & 62 & 87 \\
\hline
\end{tabular}

DRE: digital rectal examination, HRM: high resolution manometry, ARM: anorectal manometry, DEF: defecography, EMG: electromyography, and CCT: colonic transit time.

DRE: as-pc (anal sphincter paradoxical contraction), as-nr (anal sphincter nonrelaxing), am-nc (abdominal muscles not contracted), pd-a (perineal descent absent), and pe-i (push effort impaired).

HRM or ARM: pr-pc (puborectalis paradoxical contraction) or pr-nr (puborectalis nonrelaxing).

DEF: aa-ni (anorectal angle not increased).

EMG: i (activity increased).

CTT: stt (slow transit time).

BET: ne (not expelling a $50 \mathrm{~mL}$ water balloon in 1 minute).

$64-90 \%)$ and the pooled specificity was $84 \%$ (CI 64-94\%). Table 3 reviews the data regarding the DRE.

When we consider only studies using Rome criteria for CC $[28,36]$, the sensitivity was $78 \%$ and the specificity was $90 \%$. When we repeated the analysis with studies with precise DRE diagnostic criteria for DD [28, 37], the sensitivity was $86 \%$ and the specificity was $72 \%$.

The pooled NPV of the DRE was $64 \%$ (CI 37-85\%).

\section{Discussion}

Nowadays, when medicine faces new challenges concerning cost-effectiveness, a cheaper but satisfactory diagnostic approach of DD that promotes an adequate selection of complementary tests and an earlier and targeted treatment seems ideal. This article intends to follow a creative approach to the subject "diagnosis of the DD" with the low-cost tests 


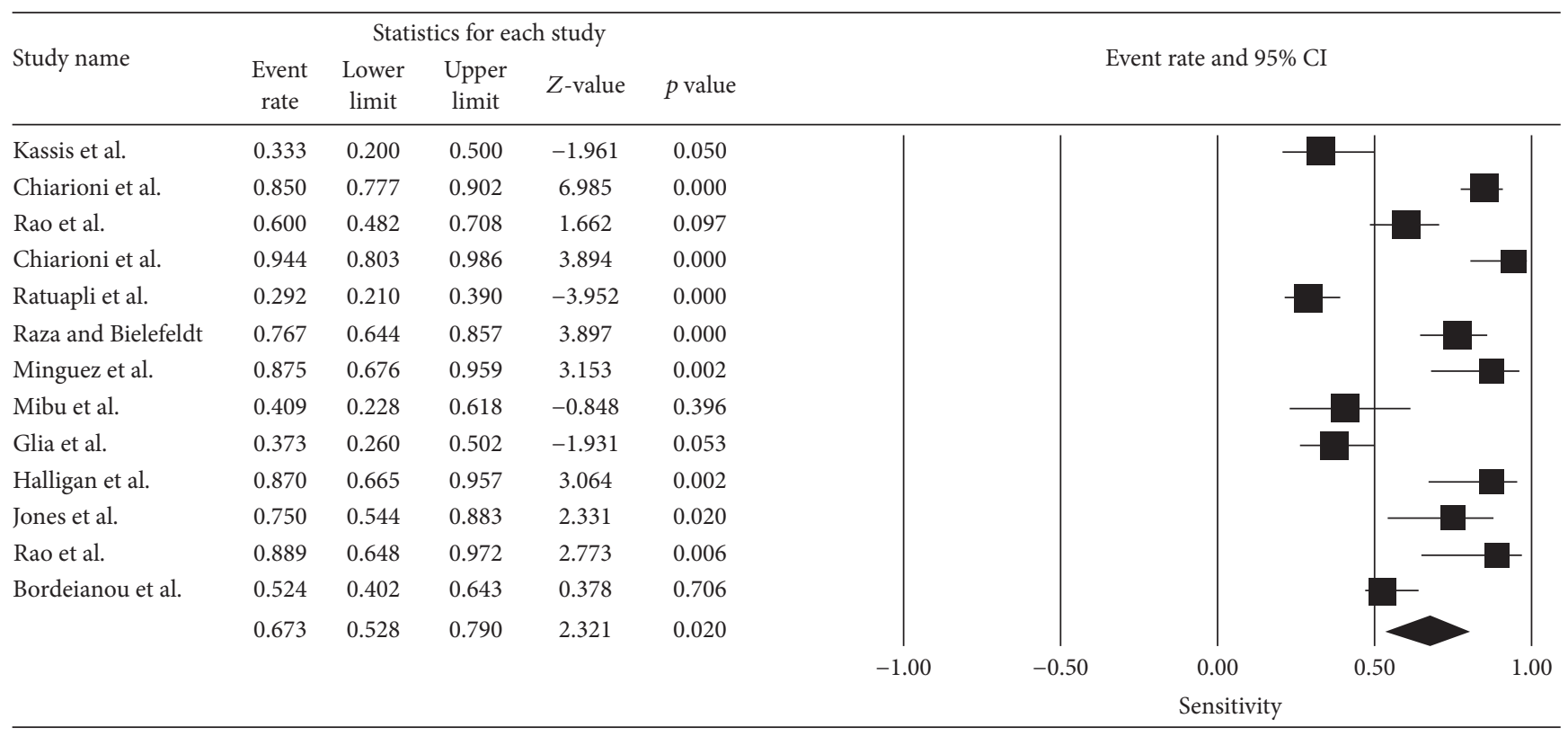

Meta-analysis

FIGURE 2: Summary measures for sensitivity of BET analysis.

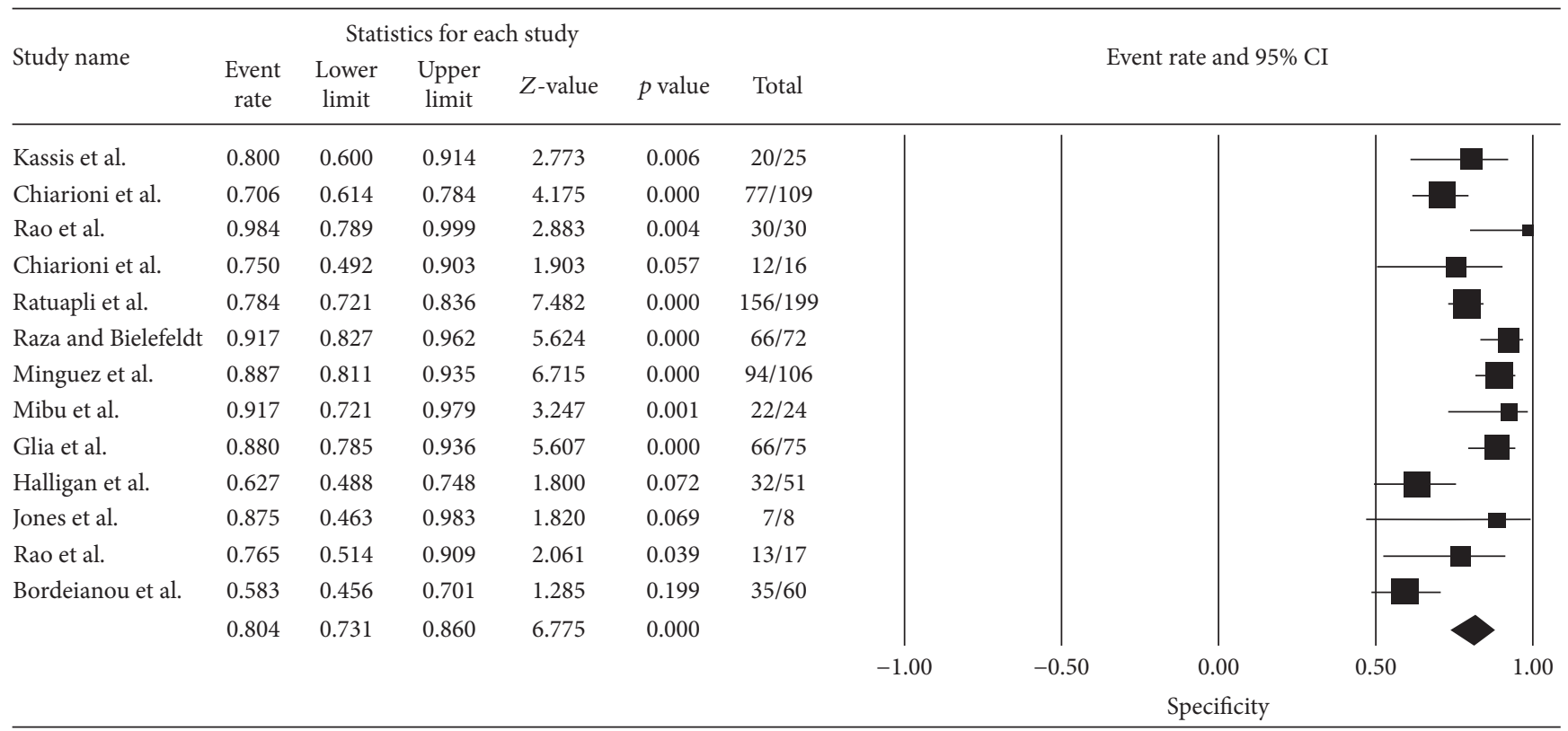

Meta-analysis

FIGURE 3: Summary measures for specificity of BET analysis.

evaluated as screening or excluding options in patients with CC. With this idea in mind, we were able to systematically gather a vast collection of data related to the BET and the DRE.

Previous reviews $[22,38]$ collected important data related to all anorectal physiological tests and faced diverse challenges: variable diagnostic criteria, different protocols of physiological tests, and lack of definitions for positive results. As expressed in Tables 2 and 3, we also faced heterogeneous protocols and variable diagnostic criteria in each centre; on the other hand, we recognize an increased effort to define negative and positive results.

Considering the data from the 17 selected articles, this meta-analysis evaluated a total of 2329 patients and calculated 
a pooled sensitivity and specificity for the BET of $67 \%$ and $80 \%$, respectively, and a pooled sensitivity and specificity for the DRE of $80 \%$ and $84 \%$, respectively.

As the BET technique was not standardized, the included articles described different methodologies [33, 34, 39-43]. Most studies of this meta-analysis described the use of the seated position (12 studies) probably because this method better resembles the act of defecation. Excluding the studies that filled the balloon with air [13,41, 42], the sensitivity and the specificity of the water-filled BET did not change. Studies that evaluated different sizes and consistencies of simulated stools found that small and hard stools are harder to expel than large softer stools $[17,44]$. Although probably air simulates inadequately the stool weight and consistency, we did not see any difference in sensitivity and specificity, perhaps because of the low number of patients evaluated (301 out of 1450). Another aspect to take into account is the volume of the balloon-the majority of the studies reported fixed volumes in their series but the volume described varied between $10 \mathrm{~mL}$ [45] and $60 \mathrm{~mL}$ [43]; in addition, a study described a variable volume (median volume of $250 \mathrm{~mL}$ ) related to the permanent desire to evacuate [33]. When we use the cut-off of $\geq 50 \mathrm{~mL}$, the sensitivity and specificity did not change, nor when we exclude only Minguez series (median volume of $250 \mathrm{~mL}$ ). As far as we know, no study compared fixed and variable volumes on the testing protocol of the BET.

Also most studies do not report the balloon materialsilicone or latex-that also influences the volume associated with first sensation, urgency, and maximum discomfort [31]. Perhaps with a larger number of patients and a prospective and controlled comparison between different volumes and materials, a more correct performance of the BET in terms of sensitivity and specificity is possible. The time given to the patient to evacuate the balloon also varied between 1 [33] and 5 minutes $[5,13,35]$. When we use the cut-off of 2 minutes as the limit between normal and abnormal BET as proposed by Chiarioni et al. [34], the pooled sensitivity and specificity rose to $74 \%$ and $84 \%$, respectively.

The majority of the studies reviewed in this meta-analysis were anterior to 2014 and did not define the cut-off time for normal/abnormal BET or use higher cut-off values. Probably the use of a consistent and low cut-off time for the BET in future studies will improve the value of the test. Due to all these issues, studies that use a standardized methodology of the BET, including balloon volume, are necessary. Standardization of the BET is relevant not only to diagnosis but also to treatment [46].

The analysis of the comparative anorectal test showed us that the pooled sensitivity and specificity of the BET is equivalent to ARM, but it is lower than the pooled sensitivity and specificity of the defecography. It is higher when compared to the pooled sensitivity and specificity of the combination of the tests. The consistency of results in the subanalysis of ARM is easily explained by the fact that both the BET and the ARM are purely physiological tests while defecography has also a morphological component assessment which makes it not so suitable to use as comparative test. Only two studies $[33,35]$ used a combination of tests to diagnose DD which may underestimate the prevalence of dyssynergia in their study population. However considering that Rome III criteria also recommend at least two morphological or physiological abnormalities to diagnose DD (in one or more tests), the BET performance comparing to a combination of tests is probably more reliable. Although we described a range of possible explanations for the moderate sensitivity and specificity of the BET compared to the "classic" physiological tests, the large overlap between normal subjects and patients with symptoms may also be explained by the BET being an unphysiological maneuver.

Although guidelines highlight the importance of rectal examination for identifying DD [6], we only collected 4 studies evaluating DRE that met our inclusion criteria. The pooled sensitivity and specificity were 80 and $84 \%$, respectively. The small number of studies along with the heterogeneity revealed by the meta-analysis prevents us from drawing any conclusion from these data. When we evaluate only the studies that use Rome criteria [28, 36], the sensitivity and the specificity were comparable. None of the selected studies used the DRESS published by Orkin et al. in 2010 [29]. Tantiphlachiva et al. [28] and Soh et al. [37] described their own diagnostic criteria for DD using DRE, while two earlier studies $[36,47]$ used expert self-reported evaluation. When we analyse only the studies with more strict criteria, the sensitivity rose to $86 \%$ and the specificity decreased to $72 \%$. Although we cannot take any definitive conclusion from this small number of patients, a possible explanation is that even less skilled practitioners can be aware of the possible abnormalities in anorectal examination when more descriptive and explanatory criteria are used. When strict criteria are used, the decreased specificity is probably related to the exclusion of knowledge of the experts in the diagnosis of DD using the DRE. We cannot deny the subjectivity implied in a physical examination-the question is if we can use it with sufficient know-how in benefit of the patient. Probably with more studies, eventual validation, and widespread use of the DRESS or Tantiphlachiva criteria, we will be capable of understanding the exact role of the DRE in diagnosing DD. It was not possible to run a subanalysis based on the comparative anorectal test because all studies except one [37] used a combination of symptoms and/or exams.

The NPV of the BET and the DRE was $72 \%$ and $64 \%$, making these tests unsuitable for excluding the diagnosis of DD in constipated patients.

This meta-analysis has several limitations. There was no enough consistency among studies, which influenced our estimated results. The criteria to define CC were variable, from not defined or self-reported to Rome III criteria. The lack of standardization of the BET protocols made the comparison of studies problematic. Also the absence of a true comparative gold-standard test or unique diagnostic criteria for DD makes any review on the subject a difficult task. At last, another limitation, inherent to all anorectal physiological tests, is the imperfect simulation of the act of defecation due to the influence of laboratory conditions.

In conclusion, with current data, although the importance of the BET and the DRE in the investigation of constipated patients is obvious, so far we cannot use these low-cost tests with a screening or exclusion purpose. There is a need 
for prospective studies with descriptive and consistent methodology to evaluate its utility and cost-effectiveness and confirm its reproducibility in DD diagnostic diagram. For now, the results presented in this manuscript demonstrate that the BET and the DRE should be interpreted alongside the results of the other tests of anorectal function.

\section{Competing Interests}

The authors declare that they have no competing interests.

\section{References}

[1] J. Noelting, J. Eaton, P. Thapa, A. R. Zinsmeister, G. R. Locke, and A. E. Bharucha, "Sa2035 incidence rate and characteristics of clinically diagnosed defecatory disorders in the community," Gastroenterology, vol. 144, no. 5, pp. S-365-S-366, 2013.

[2] D. M. Preston and J. E. Lennard-Jones, "Anismus in chronic constipation," Digestive Diseases and Sciences, vol. 30, no. 5, pp. 413-418, 1985.

[3] S. S. C. Rao, K. D. Welcher, and J. S. Leistikow, "Obstructive defecation: a failure of rectoanal coordination," The American Journal of Gastroenterology, vol. 93, no. 7, pp. 1042-1050, 1998.

[4] M. A. Gladman, P. J. Lunniss, S. M. Scott, and M. Swash, "Rectal hyposensitivity," American Journal of Gastroenterology, vol. 101, no. 5, pp. 1140-1151, 2006.

[5] G. Chiarioni, L. Salandini, and W. E. Whitehead, "Biofeedback benefits only patients with outlet dysfunction, not patients with isolated slow transit constipation," Gastroenterology, vol. 129, no. 1, pp. 86-97, 2005.

[6] A. E. Bharucha, J. H. Pemberton, and G. R. Locke III, "American gastroenterological association technical review on constipation," Gastroenterology, vol. 144, no. 1, pp. 218-238, 2013.

[7] A. E. Bharucha and S. S. C. Rao, "An update on anorectal disorders for gastroenterologists," Gastroenterology, vol. 146, no. 1, pp. 37-45.e2, 2014.

[8] G. F. Longstreth, W. G. Thompson, W. D. Chey, L. A. Houghton, F. Mearin, and R. C. Spiller, "Functional bowel disorders," Gastroenterology, vol. 130, no. 5, pp. 1480-1491, 2006.

[9] J. Rai, W. D. Chey, M. Maneerattanaporn, J. Baker, and R. J. Saad, "Tu2013 do constipation symptoms identify patients with dyssynergic defecation?" Gastroenterology, vol. 142, no. 5, supplement 1, article S-901, 2012.

[10] A. L. Halverson and B. A. Orkin, "Which physiologic tests are useful in patients with constipation?" Diseases of the Colon and Rectum, vol. 41, no. 6, pp. 735-739, 1998.

[11] S. S. C. Rao, R. S. Mudipalli, M. Stessman, and B. Zimmerman, "Investigation of the utility of colorectal function tests and Rome II criteria in dyssynergic defecation (Anismus)," Neurogastroenterology \& Motility, vol. 16, no. 5, pp. 589-596, 2004.

[12] S. S. C. Rao and K. Meduri, "What is necessary to diagnose constipation?" Best Practice and Research: Clinical Gastroenterology, vol. 25, no. 1, pp. 127-140, 2011.

[13] L. Bordeianou, L. Savitt, and A. Dursun, "Measurements of pelvic floor dyssynergia: which test result matters?" Diseases of the Colon and Rectum, vol. 54, no. 1, pp. 60-65, 2011.

[14] P. N. Jones, D. Z. Lubowski, M. Swash, and M. M. Henry, "Is paradoxical contraction of puborectalis muscle of functional importance?" Diseases of the Colon \& Rectum, vol. 30, no. 9, pp. 667-670, 1987.
[15] W. A. Voderholzer, D. A. Neuhaus, A. G. Klauser, K. Tzavella, S. A. Müller-Lissner, and N. E. Schindlbeck, "Paradoxical sphincter contraction is rarely indicative of anismus," Gut, vol. 41, no. 2, pp. 258-262, 1997.

[16] S. S. C. Rao, R. Hatfield, E. Soffer, S. Rao, J. Beaty, and J. L. Conklin, "Manometric tests of anorectal function in healthy adults," The American Journal of Gastroenterology, vol. 94, no. 3, pp. 773-783, 1999.

[17] S. S. C. Rao, R. Kavlock, and S. Rao, "Influence of body position and stool characteristics on defecation in humans," The American Journal of Gastroenterology, vol. 101, no. 12, pp. 27902796, 2006.

[18] M. T. Eltringham, U. Khan, I. M. Bain et al., "Functional defecation disorder as a clinical subgroup of chronic constipation: analysis of symptoms and physiological parameters," Scandinavian Journal of Gastroenterology, vol. 43, no. 3, pp. 262-269, 2008.

[19] A. E. Bharucha, J. G. Fletcher, B. Seide, S. J. Riederer, and A. R. Zinsmeister, "Phenotypic variation in functional disorders of defecation," Gastroenterology, vol. 128, no. 5, pp. 1199-1210, 2005.

[20] I. J. Cook, N. J. Talley, M. A. Benninga, S. S. Rao, and S. M. Scott, "Chronic constipation: overview and challenges," Neurogastroenterology \& Motility, vol. 21, supplement 2, pp. 1-8, 2009.

[21] G. S. Duthie and D. C. C. Bartolo, "Anismus: the cause of constipation? Results of investigation and treatment," World Journal of Surgery, vol. 16, no. 5, pp. 831-835, 1992.

[22] S. S. C. Rao, R. Ozturk, and L. Laine, "Clinical utility of diagnostic tests for constipation in adults: a systematic review," American Journal of Gastroenterology, vol. 100, no. 7, pp. 1605-1615, 2005.

[23] V. K. Dik, P. D. Siersema, A. Joseph, P. Hodgkins, H. M. Smeets, and M. G. H. Van Oijen, "Constipation-related direct medical costs in 16887 patients newly diagnosed with chronic constipation," European Journal of Gastroenterology and Hepatology, vol. 26, no. 11, pp. 1260-1266, 2014.

[24] P. C. Rantis Jr., A. M. Vernava III, G. L. Daniel, and W. E. Longo, "Chronic constipation-is the work-up worth the cost?" Diseases of the Colon and Rectum, vol. 40, no. 3, pp. 280-286, 1997.

[25] A. Sonnenberg and T. R. Koch, "Epidemiology of constipation in the United States," Diseases of the Colon \& Rectum, vol. 32, no. 1, pp. 1-8, 1989.

[26] R. K. Wong, D. A. Drossman, A. E. Bharucha et al., "The digital rectal examination: A multicenter survey of physicians' and students' perceptions and practice patterns," American Journal of Gastroenterology, vol. 107, no. 8, pp. 1157-1163, 2012.

[27] N. J. Talley, "How to do and interpret a rectal examination in gastroenterology," The American Journal of Gastroenterology, vol. 103, no. 4, pp. 820-822, 2008.

[28] K. Tantiphlachiva, P. Rao, A. Attaluri, and S. S. C. Rao, "Digital rectal examination is a useful tool for identifying patients with dyssynergia," Clinical Gastroenterology and Hepatology, vol. 8, no. 11, pp. 955-960, 2010.

[29] B. A. Orkin, S. B. Sinykin, and P. C. Lloyd, "The digital rectal examination scoring system (DRESS)," Diseases of the Colon and Rectum, vol. 53, no. 12, pp. 1656-1660, 2010.

[30] D. A. Drossman, "Medicine has become a business, but what is the cost?" Gastroenterology, vol. 126, no. 4, pp. 952-953, 2004.

[31] J. Noelting, S. K. Ratuapli, A. E. Bharucha, D. M. Harvey, K. Ravi, and A. R. Zinsmeister, "Normal values for high-resolution anorectal manometry in healthy women: effects of age and 
significance of rectoanal gradient," American Journal of Gastroenterology, vol. 107, no. 10, pp. 1530-1536, 2012.

[32] S. S. C. Rao, "Constipation: evaluation and treatment of colonic and anorectal motility disorders," Gastroenterology Clinics of North America, vol. 36, no. 3, pp. 687-711, 2007.

[33] M. Minguez, B. Herreros, V. Sanchiz et al., "Predictive value of the balloon expulsion test for excluding the diagnosis of pelvic floor dyssynergia in constipation," Gastroenterology, vol. 126, no. 1, pp. 57-62, 2004.

[34] G. Chiarioni, S. M. Kim, I. Vantini, and W. E. Whitehead, "Validation of the balloon evacuation test: reproducibility and agreement with findings from anorectal manometry and electromyography," Clinical Gastroenterology and Hepatology, vol. 12, no. 12, pp. 2049-2054, 2014.

[35] S. S. C. Rao, K. D. Welcher, and J. S. Leistikow, "Obstructive defecation: a failure of rectoanal coordination," American Journal of Gastroenterology, vol. 93, no. 7, pp. 1042-1050, 1998.

[36] X. F. Guo, M. Y. Ke, Z. F. Wang, X. C. Fang, B. Wu, and Y. P. Tu, "Categorization of dysmotility in patients with chronic constipation and its significance for management," Chinese Journal of Digestive Diseases, vol. 5, no. 3, pp. 98-102, 2004.

[37] J. S. Soh, H. J. Lee, K. W. Jung et al., “The diagnostic value of a digital rectal examination compared with high-resolution anorectal manometry in patients with chronic constipation and fecal incontinence," The American Journal of Gastroenterology, vol. 110, no. 8, pp. 1197-1204, 2015.

[38] E. J. Videlock, A. Lembo, and F. Cremonini, "Diagnostic testing for dyssynergic defecation in chronic constipation: metaanalysis," Neurogastroenterology and Motility, vol. 25, no. 6, pp. 509-520, 2013.

[39] N. C. Kassis, J. M. Wo, T. N. James-Stevenson, D. D. T. Maglinte, M. H. Heit, and D. S. Hale, "Balloon expulsion testing for the diagnosis of dyssynergic defecation in women with chronic constipation," International Urogynecology Journal, vol. 26, no. 9, pp. 1385-1390, 2015.

[40] S. K. Ratuapli, A. E. Bharucha, J. Noelting, D. M. Harvey, and A. R. Zinsmeister, "Phenotypic identification and classification of functional defecatory disorders using high-resolution anorectal manometry," Gastroenterology, vol. 144, no. 2, pp. 314-322.e2, 2013.

[41] N. Raza and K. Bielefeldt, "Discriminative value of anorectal manometry in clinical practice," Digestive Diseases and Sciences, vol. 54, no. 11, pp. 2503-2511, 2009.

[42] R. Mibu, M. Hotokezaka, T. Kai, Y. Tanabe, and M. Tanaka, "A simplified defaecographic procedure for the assessment of faecal incontinence or obstructed defaecation," Colorectal Disease, vol. 3, no. 5, pp. 328-333, 2001.

[43] A. Glia, G. Lindberg, L. H. Nilsson, L. Mihocsa, and J. E. Åkerlund, "Constipation assessed on the basis of colorectal physiology," Scandinavian Journal of Gastroenterology, vol. 33, no. 12, pp. 1273-1279, 1998.

[44] J. J. Bannister, P. Davison, J. M. Timms, C. Gibbons, and N. W. Read, "Effect of stool size and consistency on defecation," Gut, vol. 28, no. 10, pp. 1246-1250, 1987.

[45] S. Halligan, J. Thomas, and C. Bartram, "Intrarectal pressures and balloon expulsion related to evacuation proctography," Gut, vol. 37, no. 1, pp. 100-104, 1995.

[46] B. Lestàr, F. Penninckx, and R. Kerremans, "Biofeedback defaecation training for anismus," International Journal of Colorectal Disease, vol. 6, no. 4, pp. 202-207, 1991.
[47] U. Karlbom, K. Edebol Eeg-Olofsson, W. Graf, S. Nilsson, and L. Påhlman, "Paradoxical puborectalis contraction is associated with impaired rectal evacuation," International Journal of Colorectal Disease, vol. 13, no. 3, pp. 141-147, 1998. 


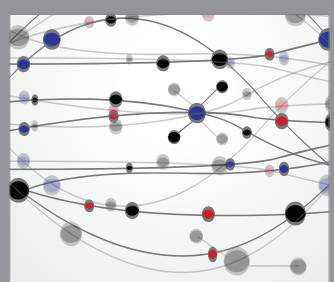

The Scientific World Journal
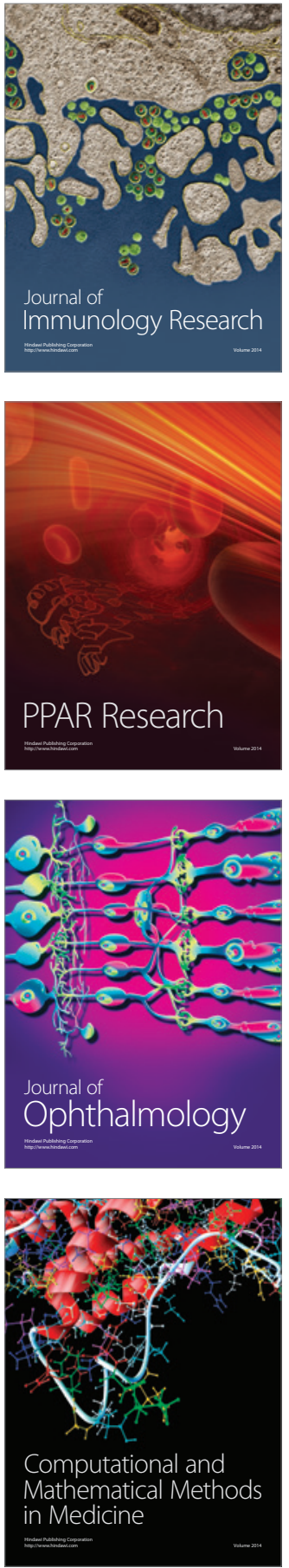

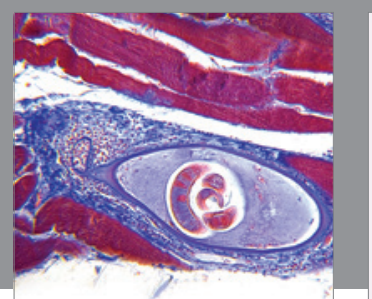

Gastroenterology Research and Practice

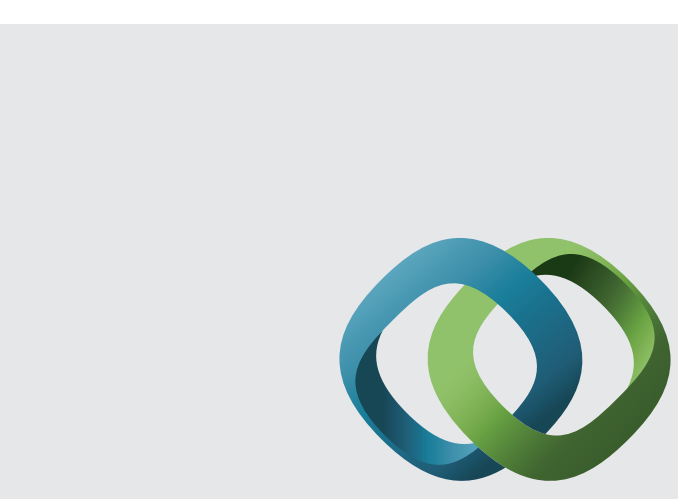

\section{Hindawi}

Submit your manuscripts at

http://www.hindawi.com
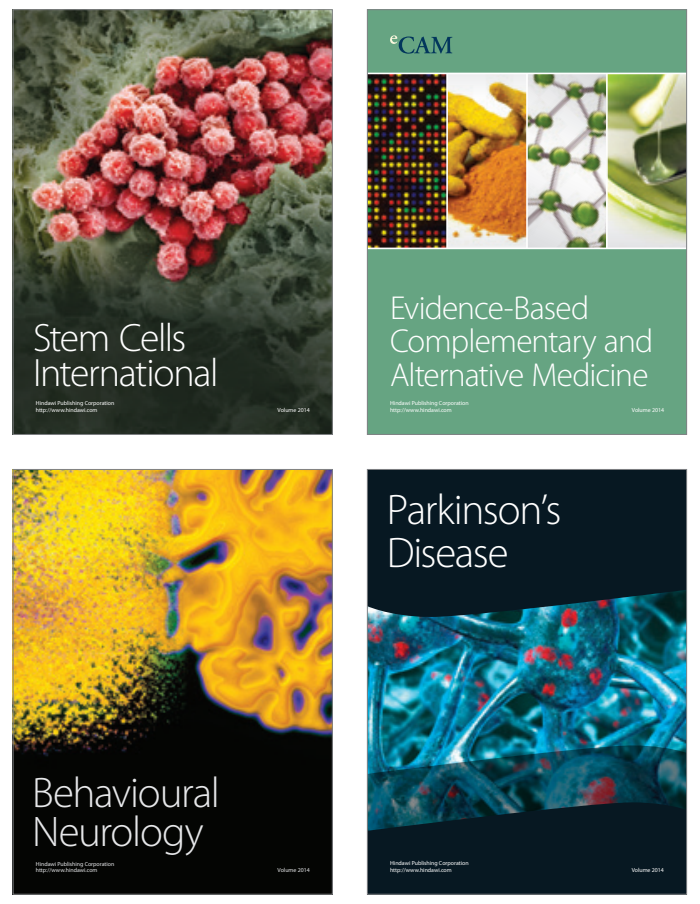
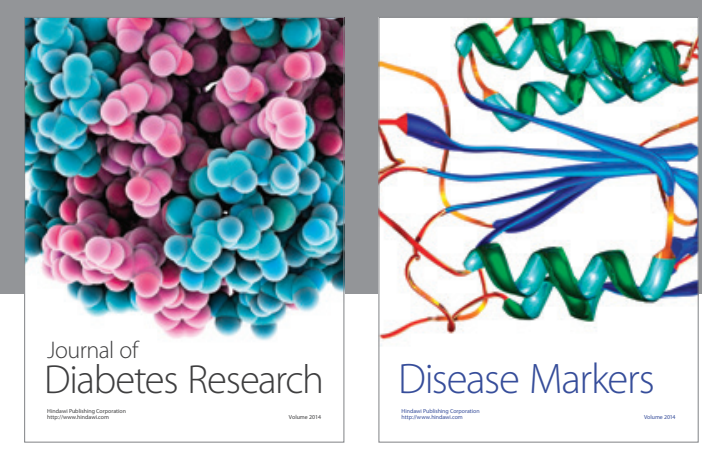

Disease Markers
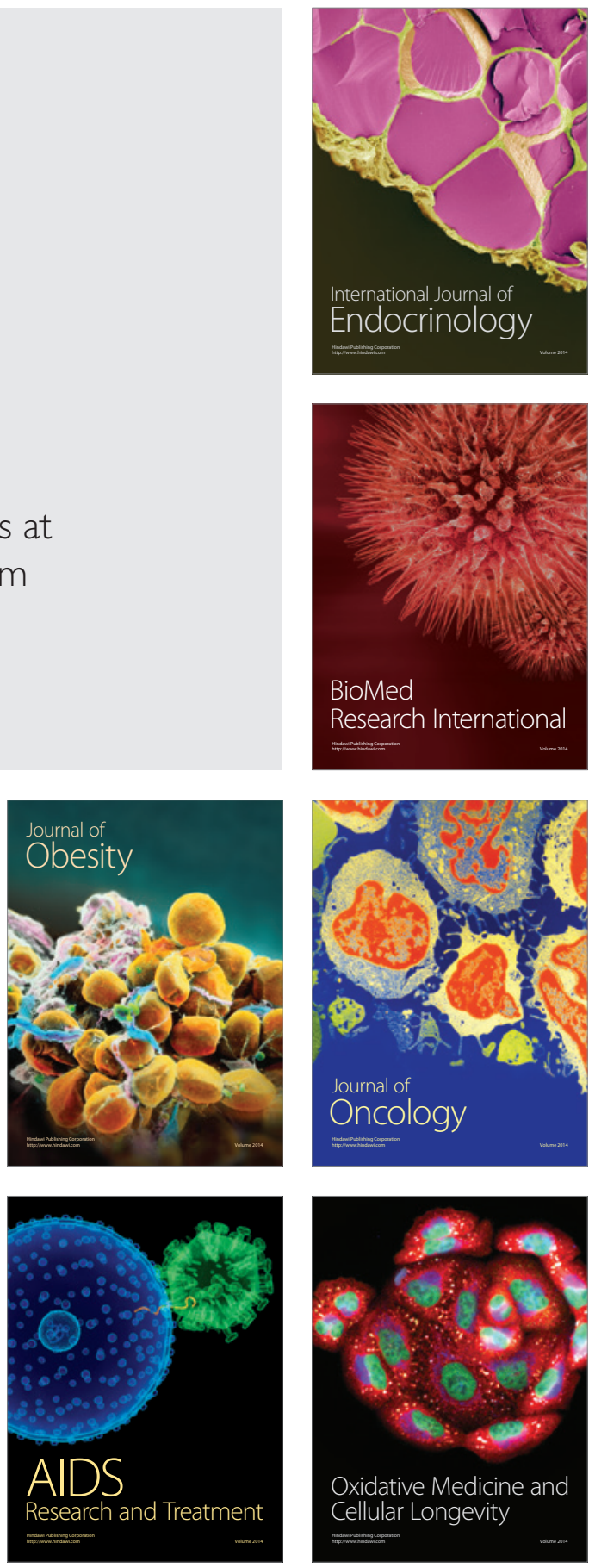